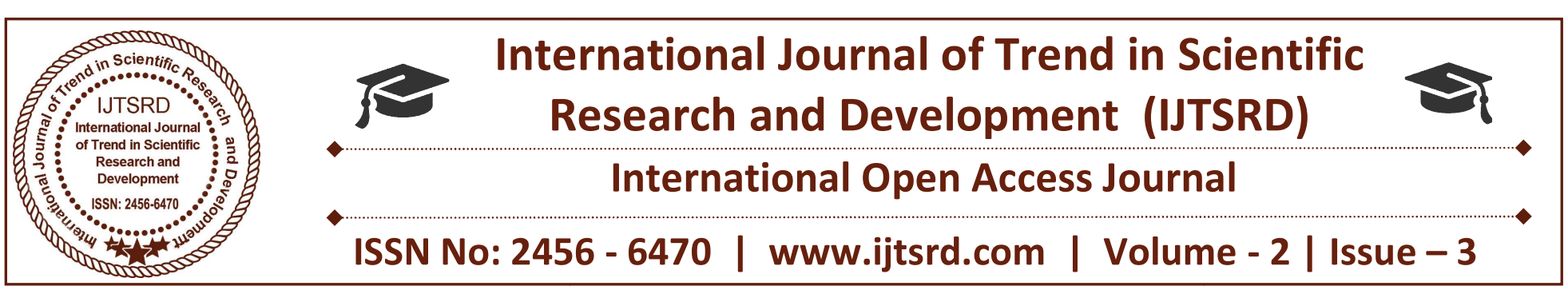

\title{
Analytic System Based on Prediction Analysis of Social Emotions from Users : A Review
}

\author{
Vaishnavi Chimote \\ Department of Computer Science and Engineering, \\ DRGIT\&R, Amravati, Maharashtra, India
}

\author{
Prof. Vrushali D. Dharmale \\ Assistant Professor, Department of Computer Science \\ and Engineering, DRGIT\&R, Amravati, \\ Maharashtra, India
}

\begin{abstract}
Over social media there are lots of symbols are used as compared to text this is an unstructured type of which get considers day by day increase in such symbols is moving the towards the new data prediction determination technique.
\end{abstract}

Due to the rapid development of Web, large numbers of documents assigned by readers' emotions have been generated through new portals. Comparing to the previous studies which focused on author's perspective, our research focuses on readers' emotions invoked by news articles. Our research provides meaningful assistance in social media application such as sentiment retrieval, opinion summarization and election prediction. In this paper, we predict the readers' emotion of news based on the social opinion network. More specifically, we construct the opinion network based on the semantic distance. The communities in the news network indicate specific events which are related to the emotions. Therefore, the opinion network serves as the lexicon between events and corresponding emotions. We leverage neighbor relationship in network to predict readers' emotions. As a result, our methods obtain better result than the state-of-the-art methods. Moreover, we developed a growing strategy to prune the network for practical application. The experiment verifies the rationality of the reduction for application.

In this paper, we implement social opinion prediction by generating a real-time social opinion network. In more details, first, we train word vectors according to the most recent Wikipedia word corpus. Second, we calculate se-mantic distance between news via word vectors. As a metric between opinions, semantic distance allows us to construct the opinions growing network to describe the dynamical social opinions. Last, we predict follow-up

Keywords: Affect sensing and analysis, recognition of group emotion, affective text mining, complex network

\section{I. alINTRODUCTION}

Social emotion prediction is of value to market analysis and to political decision[1], [2], [3], [4]. With the free and convenient communication environment of internet, people show increasing enthusiasm of online communication [5], [6].Meanwhile, the internet users prefer to pro-duce and convey online information through expressing personal opinions than just obtain online information. In this way, numerous news articles and comments have been published and shared rapidly via social media services. As a result, abundant underlying positive or negative emotion information spreads and reflects the social sentiment tendency. Most intuitively, emotional label has been widely used in social web services. Fig. 1 indicates the result of voting for a news article using emotion labels from a popular news portal. Large numbers of people concerned about a hot news online. Therefore, valuable and available emotional information is continuously pro-vided for scientific research work[7], [8], [9].Furthermore, comparing to the traditional methods, which need to do numbers of surveys offline, data processing technology has been 
developed more feasible in the field of emotion-al extraction, analysis and prediction with its benefits of lower cost, higher efficiency and more accuracy. Under this circumstance, readers' emotions prediction shows a highly research potential.

Compared with the typical tasks of sentiment analysis, opinion mining or affect recognition which based on subjective text, social opinion prediction focuses on objective text, for example news articles, which may not contain any opinion, but can evoke readers' certain emotion. Due to the particularity of the task, social opinion prediction has potential applications which are different from those of writer-sentiment analysis[10]. Considering the effect of social media on the public sentiment, social emotion analysis engenders large benefits to social and economic problem, such as political issues and brand perception.

In this paper, we implement social opinion prediction by generating a real-time social opinion network. In more details, first, we train word vectors according to the most recent Wikipedia word corpus. Second, we calculate se-mantic distance between news via word vectors. As a metric between opinions, semantic distance allows us to construct the opinions growing network to describe the dynamical social opinions. Last, we predict follow-up news' social emotion based on then network.

The opinion growing network naturally incorporates the knowledge encoded in the word2vec space and leads to high performance - the predicting via networkoutper-forms all9 state-of-the-art emotion models[8],[11], [12], [13], [14], [15], [16], [17], [18], [19]. It is highly interpretable based on the opinion growing network. The opinion growing network as stereotypical knowledge grows as the follow-up news join. With the enrichment of knowledge, the prediction results become more accurate.

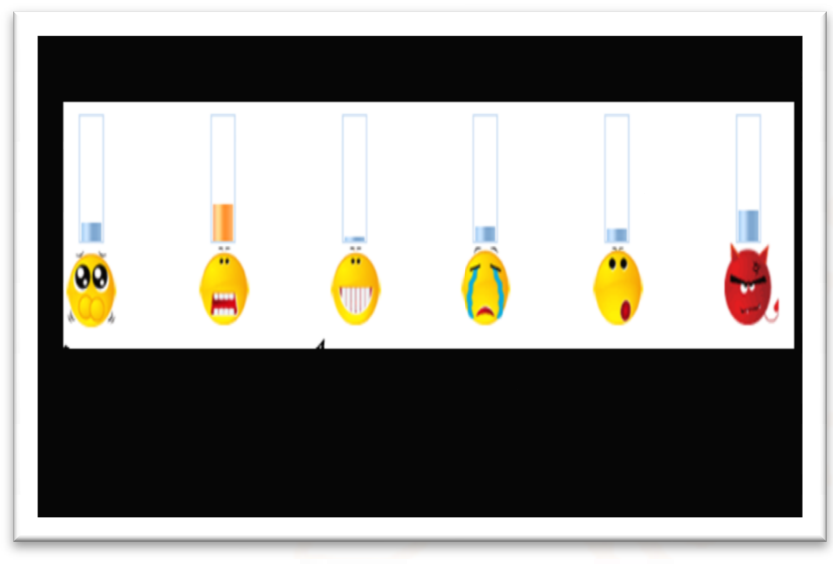

Moreover, we can leverage existing networks to provide visual summaries to analyze social emotions evolution and social hotspots.

The rest of this article is organized as follows. In Section 2, we provide related work. In Section 3, we discuss methodology and key techniques in detail. In section 4, we illustrate and discuss the dataset, experimental results. In section 5,we provide concluding remark.

\section{EXISTING WORK}

In existing paper it is proposed that the system can do the prediction of emotions of the users they are taken the reference of the news article which help us to know about the users emotions regarding to such a article .In this the experiment get proposed on datasets. Social opinion prediction is a difficult research endeavor. As the initial research work on social opinion prediction, "affective text" in SemEval2007 Tasks. Intend to annotate news headlines for the evoked emotion of readers. Another research focus on readers' emotion evoked by news sentences. Existing methods of social opinion prediction can be divided into three categories: knowledge-based techniques, statistical methods and hybrid approaches. Because of the deficiency of information of news text.it is unmanageable to annotate the emotions consistently. Knowledge-based techniques utilize existing emotional lexicon to supplement the prior knowledge for annotating the emotions. The popular emotional lexicon includes Affective Lexicon, linguistic annotation scheme, Word Net-Affect, Senti Word Net, and Sentic Net. The drawback of knowledgebased techniques is the reliance on the coverage of the emotional lexicon. These techniques cannot process terms that do not appear in the emotional lexicon. Statistical methods predict social opinion by training a 
statistical model based on a large number of welllabeled corpuses

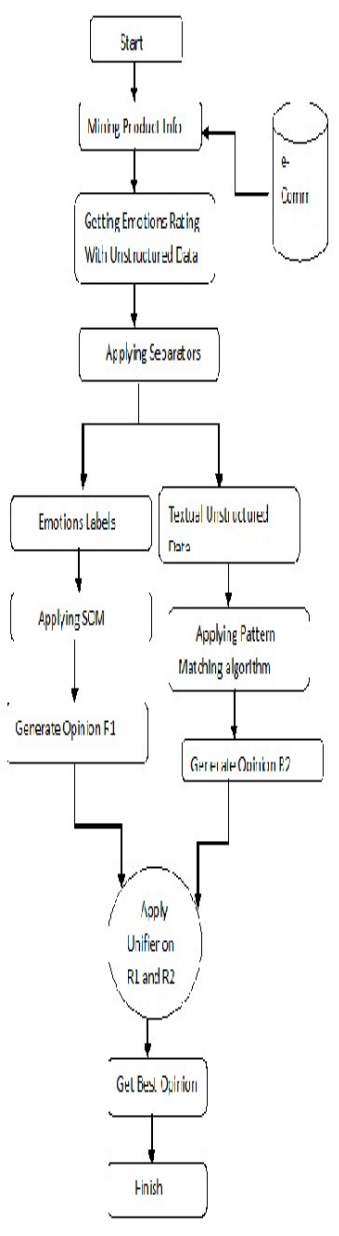

Dig: Proposed System Diagram

\section{PROBLEM DEFINITION AND ANALYSIS}

In this section, the problem about social opinion prediction Is well defined, including the relevant general terms and notations. Later on, we describe the social opinion model and opinion network in detail. Finally, we propose a strategy for social opinion prediction.

\subsection{Problem Formulation}

We define the following notations for describing the social opinion prediction: An online news collection consists of news, and the emotion ratings labels. The list of emotion labels is denoted by, and indicates emotion titled "joy", "anger", "fear", "surprise", "touching", "empathy", "boredom", "sadness", "warmness" etc. In particular, a news is a set of word tokens, and a set of ratings over $E$ emotion labels denoted by .The value of is the number of online users who have voted the $k$ th emotion label for news. According to Kim and Hovy [44], the opinion can be split into four parts: topic, opinion holder, claim, and sentiment. Tobespecific, a holder believes a claim about a topic with a sentiment. For social opinion, the opinion holder stands for users who have voted the news. The topic can be replaced by the content of the news. The sentiment can be measured by the vote around the set of predefined emotional labels. The claim is unobservable and in essential in this task. This kind of social opinion can be model with a quadruple, where stands for the social event; is the text feature set of social event; is the result of voting towards social event which is represented as distribution over the predefined emotional labels. $t$ is the time when the social events occurred. The social opinion prediction task this paper discussed is focused on the prediction of based on the former social opinion quadruples.

\subsection{Social Opinion Model}

Sentiment-related phenomena can be explained as the process of evaluation of events, objects or persons [23], [45], [46]. The opinions are caused by the subjective evaluation of the "raw" stimuli. The "raw" stimuli may have no intrinsic emotional meaning, but will be appraised by personal relevance and implications[47]. For social opinion, the "raw" stimuli are only text and its features which are difficult to expound the corresponding sentimentrelated phenomena. In fact, there are less than $5 \%$ of directly emotional words of a text in daily speech, emotional writing, and affect-laden poetry[48]. In journalism domain, a lower percentage is undisputed. It is rarely influenced by the personal relevance under the social community. To simplify the problem, we focus on implications without personal relevance.

According to the cognitive approaches, the result of voting is "the person's experience, goals and opportunities for action"'[43]. It is process that evaluates an event by dimensions such as urgency, consistency with goals, etc. All the social opinions share the similar emotional experience, goals and opportunities for action with each other. From the NLP perspective, the models are in explicable but feasible. From psychology and linguistics perspective, the models are explicable but lack of use in the service. Based on the general characteristic, similarity 
is one of six principles that guide human perception of the worldin Gestalt theory[28], [49].We can predict social opinions by measuring the semantic similarity between events. The social cognitive process can be modeled based on a stereotypical knowledge set consisting of social opinion. Instead of establishing appraisal criteria, the cognitive process can be regarded as the neighbor analysis in set $P$. The set $P$ can be interpreted as social experience. The cognitive process can be simplified as matching the "raw" stimuli between the priori social opinions in set $P$. The social community has stabilized emotion towards specific events. It can be explained by a social psychology behavior named "stereotype" which is a fixed view of people, groups, events, institutions, or problems[50], [51], [52].Stereotype widely exists in media[50], [51]. To be more accurate, the task is modeling the relationship between current event and priori social opinions based on semantic similarity

\subsection{Opinion Network}

Based on the relation between opinions, we construct an opinion network, in which nodes indicate opinions and edges indicate relation between opinions. The opinion network acts as stereotypical knowledge which can serve as the lexicon between events and corresponding emotions. We can predict the social opinion through the net-work. To construct the opinion network, we add the edge between nodestodenote distance. Fig. 3 presents the distribution of opinions distance from a real-world data. The figures hows that the distribution of opinions distance obeys Gaussian distribution. Then we explore the relationship between network structure and social emotions. Since the opinion network here is fully connected net-works, we filter the edge to visualize the network structure. We prune edges shorter than threshold and label the nodes of 8 emotions by color. The network structure is different in thresholds. Here we choose the threshold for the visualization of the network. The threshold is chosen as 0.7 manually for visualization of network. We utilize Force Atlas2 [54] algorithm to arrange the layout of nodes. The network shows in Fig. 4.The color of the nodes denotes the most voted emotion label in each news. The weights of edges reflect the value of distance.

\subsection{Social Opinion Prediction}

Although there is the correlation between emotion and community in opinions network, it cannot be utilized directly for its limitations. On the one hand, the threshold for pruning edges depends on the current network state. It is unable to determine the appropriate threshold of filter for the community structure. On the other hand, the high temporal complexity of community structure partitioning algorithm is not conducive to online application. To predict emotion conditioned on future unlabeled opinion which only contain words. We leverage the inference of community structure of opinions network and simplify it as neighbor analysis. So that, we can predict the social opinion based on the opinions growing network.

Firstly, in order to convert the distance into a standardized similarity, we are inspired by the literature [56] that transforms the uncertain distance relation of the opinion distance into a conditional probability to express the similarity. In more detail, we consider the two nodes, in the network. The node chooses node as the approaching node with the probability, considering the Gaussian distribution with node as the center point. If is closer to, then is larger. And vice versa, if the distance is far, is extremely small.

\section{Objectives}

Development of analytic algorithmic work for the business analytics

Implementation of network based semantic distance.

$>$ Implementation of Predictive decision making based on ionic patter matching

Development of business intelligence tools for predictive analysis

\section{CONCLUSIONS}

By looking towards the technique given in existing we are proposed a business intelligence analytic module based on emotion detection regarding to the product reviews based on mining with reviews, feedback, complaints given by users this will help us the user for giving the instant and fast response and which also become very proper for business development. In proposed we can implement the opinion network and emotion opinion model on the datasets retrieved from business data. Opinion prediction system will helps to predict and decision making in business intelligence. In this paper, we analyze the online social opinions and propose social opinion model for measuring similarity among news. Due to word-embedding pretrained on Wikipedia, model's stability and robustness are guaranteed and can hardly be influenced by the 
size of news data. Based on the similarity, we construct an opinion network to detect user-generated social emotion by the structures of opinion network. There is significant correlation between emotion and structures of news network as we expected. The performance of the prediction based on opinion network is more stable and accurate than existing models.

\section{REFERENCES}

1. E. Cambria, B. Schuller, Y. Xia, and C. Havasi, "New Avenues in Opinion Mining and Sentiment Analysis," IEEE Intell.Syst., vol. 28, no. 2, pp. 15-21, 2013.

2. E. Cambria, B. Schuller, Y. Xia, and B. White, "New avenues in knowledge bases for natural language processing," Knowledge-Based Syst., vol. 108, pp. 1-4, Sep. 2016.

3. E. Cambria, "Affective Computing and Sentiment Analysis," IEEE Intell. Syst., vol. 31, no. 2, pp. 102-107, Mar. 2016.

4. E. Cambria, N. Howard, Y. Xia, and T.-S.Chua, "Computational Intelligence for Big Social Data Analysis [Guest Editorial]," IEEE Comput.Intell. Mag., vol. 11, no. 3, pp. 8-9, Aug. 2016.

5. B. Zhang, X. Guan, M. J. Khan, and Y. Zhou, "A time-varying propagation model of hot topic on BBS sites and Blog networks," Inf. Sci. (Ny)., vol. 187, pp. 15-32, 2012.

6. Z. Sun, Q. Peng, J. Lv, and J. Zhang, "A prediction model of post subjects based on information lifecycle in forum," Inf. Sci. (Ny)., vol. 337, pp. 59-71, 2016.

7. S. Baoet al., "Joint emotion-topic modeling for social affective text mining," in Proceedings IEEE International Conference on Data Mining, ICDM, pp. 699-704, 2009.

8. S. Baoet al., "Mining Social Emotions from Affective Text," IEEE Trans. Knowl. Data Eng., vol. 24, no. 9, pp. 1658-1670, Sep. 2012.

9. Q. Wang, O. Wu, W. Hu, J. Yang, and W. Li, "Ranking social emotions by learning listwise preference," in 1st Asian Conference on Pattern Recognition, ACPR 2011, pp. 164-168, 2011.

10. K. H.-Y. Lin and H.-H.Chen, "Ranking reader emotions using pairwise loss minimization and emotional distribution regression," Proc. Conf.
Empir.Methods Nat. Lang. Process. - EMNLP '08, no. October, pp. 136-144, 2008.

11. P. Katz, M. Singleton, and R. Wicentowski, "SWAT-MP: The SemEval-2007 Systems for Task 5 and Task 14," in Proceedings of the 4th International Workshop on Semantic Evaluations, pp. 308--313, 2007.

12. Y. Rao, "Contextual Sentiment Topic Model for Adaptive Social Emotion Classification," IEEE Intell. Syst., vol. 31, no. 1, pp. 41-47, Jan. 2016.

13. Y. Rao, Q. Li, X. Mao, and L. Wenyin, "Sentiment topic models for social emotion mining," Inf. Sci. (Ny)., vol. 266, pp. 90-100, May 2014.

14. Y. Rao, Q. Li, L. Wenyin, Q. Wu, and X. Quan, "Affective topic model for social emotion detection," Neural Networks, vol. 58, no. 2012, pp. 29-37, Oct. 2014.

15. S. Poria, E. Cambria, D. Hazarika, and P. Vij, "A Deeper Look into Sarcastic Tweets Using Deep Convolutional Neural Networks," Coling 2016, pp. 1601-1612, Oct. 2016.

16. S. Aral and D. Walker, "Identifying social influence in networks using randomized experiments," IEEE Intell.Syst., vol. 26, no. 5, pp. 91-96, 2011.

17. X. Li, J. Ouyang, and X. Zhou, "Supervised topic models for multi-label classification," Neurocomputing, vol. 149, no. PB, pp. 811-819, 2015.

18. Y. Kim, "Convolutional Neural Networks for Sentence Classification," in EMNLP, vol. 21, no. 9, pp. 1746-1751, 2014.

19. S. Poria, E. Cambria, and A. Gelbukh, "Deep Convolutional Neural Network Textual Features and Multiple Kernel Learning for Utterance-level Multimodal Sentiment Analysis," in EMNLP, no. September, pp. 2539-2544, 2015. 